\title{
Patient characteristics as a moderator of post- traumatic stress disorder treatment outcome: combining symptom burden and strengths
}

\author{
Marylene Cloitre, Eva Petkova, Zhe Su and Brandon Weiss
}

\section{Background}

Post-traumatic stress disorder (PTSD) psychotherapy research has failed to identify patient characteristics that consistently predict differential outcome.

\section{Aims}

To identify patient characteristics associated with differential outcome via a statistically generated composite moderator among women with childhood abuse-related PTSD in a randomised controlled trial comparing exposure therapy, skills training and their combination.

\section{Method}

Six baseline patient characteristics were combined in a composite moderator of treatment effects for PTSD symptoms across the three treatment conditions through a 6-month follow-up.

\section{Results}

The optimal moderator was the combined burden of all symptoms and emotion regulation strength. Those with high moderator scores, reflecting high symptom load relative to emotion regulation, did least well in exposure, moderately well in skills and best in the combination.

\section{Conclusions}

A clinically meaningful moderator, which combines patient symptom burden and strengths, was identified. Assessment at follow-up may provide a more accurate indicator of variability in outcome than that obtained immediately post-treatment.

\section{Declaration of interest}

None.

\section{Copyright and usage}

(C) The Royal College of Psychiatrists 2016. This is an open access article distributed under the terms of the creative Commons Non-Commercial, No Derivatives (CC BY-NC-ND) licence.
Post-traumatic stress disorder (PTSD) is a highly prevalent psychiatric disorder ${ }^{1}$ marked primarily by symptoms of intrusions, avoidance, negative cognitions and affect, and hyperarousal following a traumatic event. ${ }^{2,3}$ It is an important public health problem associated with substantial functional impairment ${ }^{4}$ and poor quality of life. ${ }^{5}$ Current treatment recommendations for PTSD support the use of trauma-focused cognitive-behavioural therapies. ${ }^{6,7}$ However, PTSD is a highly heterogeneous disorder and typically presents with comorbid disorders and symptoms. ${ }^{8} \mathrm{~A}$ substantial proportion of patients who complete treatment still retain their diagnosis, ${ }^{9}$ and drop-out rates are substantial. ${ }^{10}$ Identification of moderators of treatment effectiveness is crucial to guiding treatment ${ }^{11}$ so as to optimise clinical outcomes and more broadly to align with patient-centred care and 'precision medicine'. ${ }^{12}$

PTSD treatment guidelines have suggested that individuals who are exposed to prolonged and repeated trauma such as childhood abuse, domestic violence and organised political violence may benefit from enhanced cognitive-behavioural therapy that includes not only trauma-focused interventions but also stress management, social support and related interventions. ${ }^{6,13}$ These populations are known to be at risk for a greater diversity and severity of symptoms beyond those of PTSD,${ }^{14}$ and it has been proposed that interventions which address comorbidities may improve outcomes.

However, investigations of baseline patient characteristics as predictors of treatment outcome have produced inconsistent results. For example, commonly occurring comorbid symptoms such as depression, dissociation and anger have been found to reduce good outcome in some studies ${ }^{15-17}$ but not in other studies. ${ }^{18}$
Different results regarding moderation have been obtained even when the same treatment has been used but in different populations. For example, shame was found to predict outcome in exposure therapy in a college clinic but not in a community clinic sample. ${ }^{17}$ In addition, some studies have shown that trauma history such as childhood abuse predicts less beneficial outcome, ${ }^{19}$ whereas others report findings that have been mixed or nil. ${ }^{17,20}$ This has led some researchers to conclude that the use of enhanced treatment is unnecessary since there is no evidence that any specific patient characteristic is associated with suboptimal outcome. ${ }^{21}$

However, it is problematic to make conclusions from nil results. The absence of differential outcomes has many possible sources. First, most of the above PTSD studies have limited their predictor analyses to outcomes at immediately post-treatment. A recent meta-analysis has reported that differences in outcomes across PTSD psychotherapies were observed between 1 and 4 months post-treatment but not at immediately post-treatment, ${ }^{22}$ suggesting that variation in the benefits of PTSD psychotherapy may be more likely to emerge during follow-up.

Second, experts in moderator analyses have noted that individual patient characteristics are likely to be weak moderators of treatment effects and that it may be necessary to combine individual weak measures to create a single strong moderator to predict differential outcome across treatments. ${ }^{23}$ Analytical methods for personalised medicine and identification of patientspecific optimal treatment decisions have been successful in physical health disorders. ${ }^{24}$ Recently, new statistical methodologies for combining baseline patient characteristics with the goal of obtaining strong moderators of treatment effects have been developed for psychiatric disorders as well. ${ }^{25,26}$ 
In this paper we utilised a generated effect modifier (GEM) approach $^{26}$ to produce an optimal moderator of the effects of three psychotherapies tested in a randomised controlled trial (RCT) of cognitive-behavioural treatment for PTSD related to childhood abuse. ${ }^{27}$ The RCT evaluated the effect of treatment on improving PTSD outcome by providing a skills-focused module to address comorbid problems related to emotion regulation and interpersonal difficulties in addition to trauma-focused (exposure) therapy. The study compared the outcomes of the skills plus exposure condition relative to an exposure without skills condition and to a skills without exposure condition. Results indicated that the skills plus exposure condition provided greater rates of PTSD remission relative to the exposure condition, whereas the skills group fell in the middle.

Here we investigated whether baseline measures or a combination of them would moderate the effect of treatment on the PTSD outcome. The goal was to identify sub-populations of patients that might benefit more from one treatment approach than the others.

\section{Method}

\section{Study design}

Data for the current study come from an RCT evaluating a twomodule treatment, Skills Training in Affective and Interpersonal Regulation (STAIR) followed by a modified form of prolonged exposure (EXP) compared with each of its component parts. The comparison conditions had one of the components eliminated and replaced with supportive counselling (SupC) to control for treatment duration, session number and therapist contact. The three treatment conditions were STAIR/EXP, STAIR/SupC and SupC/ EXP (see previous publication ${ }^{27}$ for full details of the protocol). Ethical approval for the clinical trial from which these data are drawn was obtained.

\section{Sample}

Participants were 104 women with a principal diagnosis of DSMIV-defined PTSD, diagnosed with the Clinician-Administered PTSD Scale (CAPS), ${ }^{28}$ related to childhood sexual and/or physical abuse occurring before the age of 18. Exclusion criteria were current psychotic symptoms, untreated bipolar disorder, substance dependence not in remission for at least 3 months, significant cognitive impairment and acute suicidality in the previous 3 months. Participants were randomly assigned to one of the three conditions. All participants signed written informed consent approved by the institutional review board of the university at which the study was conducted.

Participants ranged in age from 22 to 61 (mean 36.48, s.d.=9.39) and were well educated (87.4\% completing at least some college education). Ethnic distribution was as follows: 37 White (35.6\%), 29 Black (27.9\%), 27 Hispanic (26.0\%), 3 Asian (2.9\%) and 8 'Other' (7.7\%). With respect to occupational status, 32 were employed full time (30.8\%), 27 employed part-time (26.0\%), 17 unemployed
(16.3\%), 6 enrolled as students (5.8\%), 3 homemaker (2.9\%), 3 unpaid volunteer (2.9\%), 2 disabled (1.9\%) and 1 retired (1.0\%). The majority of participants were single $(n=48,46.2 \%)$, with the remainder either married ( $n=20,19.2 \%)$, living with a significant other $(n=18,17.3 \%)$, separated $(n=16,15.4 \%)$ or widowed $(n=2,1.9 \%)$.

\section{Measures}

\section{Baseline predictors}

Seven baseline predictors were considered as potential moderators of treatment effects: (1) PTSD symptom severity, assessed via the CAPS ${ }^{28}$ (2) depressive symptoms, assessed by the Beck Depression Inventory-II; ${ }^{29}$ (3) dissociation, assessed by the dissociation subscale of the Trauma Symptoms Inventory; ${ }^{30}(4)$ interpersonal problems, assessed by the Inventory of Interpersonal Problems; ${ }^{31}$ (5) anger expression, assessed by the State-Trait Anger Expression Inventory; ${ }^{32}(6)$ negative mood regulation, assessed via the Negative Mood Regulation Scale (NMR). ${ }^{33}$ See Table 1 for a description of these variables at baseline.

\section{Outcome}

PTSD symptom severity, assessed via CAPS, served as the primary outcome variable. The CAPS was conducted by independent assessors masked to treatment condition at pre-treatment, posttreatment, 3-month follow-up and 6-month follow-up evaluation sessions. See supplementary Table DS1 for means and standard deviations of CAPS scores at all time points.

\section{Statistical analyses}

First we conducted standard moderator analysis (details are provided in the data supplement). We modelled the CAPS scores at immediately post-treatment, 3- and 6-month follow-up as a function of treatment, time, a given baseline patient characteristic (the six covariates shown in Table 1) and all two- and three-way interactions between them and assessed the significance of the three-way interaction (treatment $\times$ covariate $\times$ time). Mixed effects models for longitudinal data were employed to fit those models. ${ }^{34}$ The baseline covariates were investigated one by one.

We next conducted analyses that allowed the generation of a composite moderator. Of all possible linear combinations of the six baseline covariates we identified the linear combination that resulted in the most significant three-way interaction (treatment $x$ linear combination $\times$ time) in the standard mediator model. We call this linear combination a GEM as it is generated to possess certain characteristics (i.e. moderation) rather than being an $a$ priori specified measure. ${ }^{26}$ After optimising the selected criteria, the statistical significance of the GEM as a treatment effect modifier (i.e. the $P$-value for the interaction terms) was obtained by a permutation test. (See the data supplement for details.) The computations were performed in $\mathrm{R}^{35}$

\begin{tabular}{|c|c|c|c|c|c|c|}
\hline Covariate & $\begin{array}{c}\text { Number of } \\
\text { non-missing values }\end{array}$ & Mean & s.d. & $\begin{array}{l}\text { Minimum of } \\
\text { the observed values }\end{array}$ & $\begin{array}{l}\text { Maximum of the } \\
\text { observed values }\end{array}$ & $\begin{array}{l}P \text {-value of the } \\
\text { three-way interaction }\end{array}$ \\
\hline CAPS & 104 & 63.08 & 18.62 & 28 & 119 & 0.4427 \\
\hline $\mathrm{BDI}$ & 102 & 20.62 & 9.79 & 0 & 44 & 0.4501 \\
\hline TSI-DisS & 104 & 1.33 & 0.93 & 0 & 3.9 & 0.3936 \\
\hline IIP & 101 & 1.65 & 0.59 & 0.3 & 3.3 & 0.5113 \\
\hline NMR & 104 & 90.64 & 15.88 & 54 & 133 & 0.8815 \\
\hline AXEX & 103 & 31.29 & 9.71 & 13 & 59 & 0.1863 \\
\hline
\end{tabular}




\section{Results}

\section{Baseline covariates one by one as treatment effect modifiers}

Table 1 describes the study sample with respect to the six potential modifiers of treatment effect at baseline. (See supplementary Table DS2 for the correlations between these variables.) None of these individual baseline covariates was a treatment effect modifier, as indicated by the $P$-values for the three-way interaction term (treatment $\times$ covariate $\times$ time) shown in the last column of Table 1 .

\section{GEM analysis}

The GEM analysis identified the linear combination of the six baseline characteristics that produced the most significant composite treatment effect modifier, i.e. the combination resulting in the smallest $P$-value of the three-way interaction term. This interaction term (treatment $\times \mathrm{GEM} \times$ time) had an F-test statistic with a nominal $P$-value of 0.0025 ; the permutation $P$-value was 0.008 . This indicates much higher statistical significance than that of each of the individual six predictors (Table 1).

The size and direction of the coefficients (supplementary Table DS3) in the linear combination were reviewed to interpret the GEM score. Of note, the coefficient for anger expression (AxEx, the Anger Expression subscale of the Stait-Trait Anger Expression Inventory) was negative, whereas those from all the other psychopathology measures (PTSD, depression, dissociation, interpersonal problems) were positive, reflecting the fact that AxEx acted in a way opposite from these measures in relationship to PTSD outcome. In addition, we considered that anger expression was conceptually closer to the one measure of good functioning, emotion regulation (NMR), which was scored such that higher scores reflected better functioning. As a result of these observations, we reverse scored AnEx, so that like the NMR, higher scores reflected better functioning.
Table 2 Burden and emotion regulation (BER) scores presented in quartiles

\begin{tabular}{|c|c|c|c|c|}
\hline Groups & $\begin{array}{l}\text { Mean BER } \\
\text { score (s.d.) }\end{array}$ & $\begin{array}{l}\text { Symptom } \\
\text { burden (B) }\end{array}$ & $\begin{array}{l}\text { Emotion } \\
\text { regulation (ER) }\end{array}$ & $(B-E R)^{*}$ \\
\hline Level 1 & $-0.98(0.38)$ & -0.59 & -0.39 & -0.20 \\
\hline Level 2 & $-0.24(0.15)$ & -0.16 & -0.08 & -0.08 \\
\hline Level 3 & $0.24(0.15)$ & 0.14 & 0.10 & 0.04 \\
\hline Level 4 & $0.98(0.38)$ & 0.61 & 0.38 & 0.23 \\
\hline
\end{tabular}

$\mathrm{B}-\mathrm{ER}$, burden-emotion regulation.

Higher BER level and larger (positive) difference score mean that the influence of symptoms burden is stronger than that of emotion regulation. If both burden and emotion regulation had very high scores, it is possible that BER would be high whereas B-ER would be low. However, burden and emotion regulation are strongly negatively correlated so that the likelihood of both having high or low values simultaneously is very low.

Two new variables were created, one representing 'symptom burden' and the other summarising participants' 'emotion regulation'. The 'symptom burden' is a composite measure consisting of equal weights of (standardised) PTSD, depression, dissociation and interpersonal problems, whereas the 'emotion regulation' is a composite measure of equal weights of (standardised) NMR and AnEx. The generated 'symptom burden' and 'emotion regulation' variables were negatively correlated, $\rho=-0.58$.

The simple average of these two new variables, BER (an acronym for 'burden and emotion regulation'), was evaluated as a treatment effect modifier, in a similar way as the individual baseline characteristics. The $P$-value of the three-way interaction was 0.012. Although this is not as significant as the associated $P$-value for the GEM, it is still statistically highly significant, in contrast to the individual measures that form BER, and has strong interpretable value. BER was compared with the algorithmically obtained GEM; the correlation between BER and the GEM was 0.56. Table 2 presents the standardised scores for BER, symptom burden and emotion regulation, as well as a difference score (B-ER)
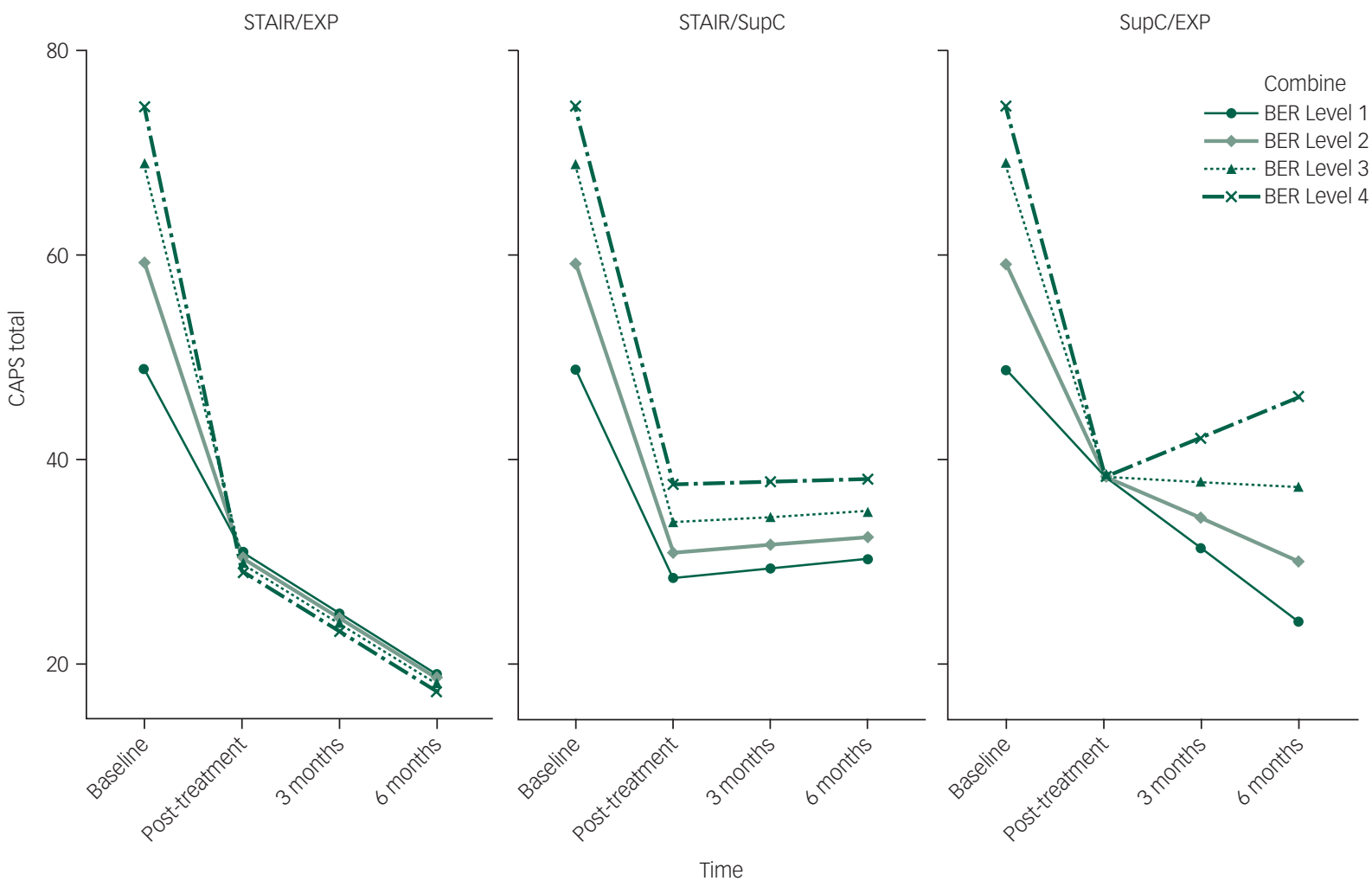
where higher scores reflect a greater burden of symptoms relative to emotion regulation.

Figure 1 depicts the course of PTSD symptoms at follow-up for four groups of patients defined by BER score quartiles. In the STAIR/EXP treatment condition, although baseline CAPS scores were different across the four BER groups such that the BER Level 1 group had the lowest CAPS score and the Level 4 group had the highest score, all participants similarly showed good outcome immediately post-treatment and experienced continuing improvement through the 6-month follow-up.

In the STAIR/SupC condition, the baseline ordering of the BER levels with respect to CAPS was preserved at all posttreatment assessments. Immediately post-treatment, participants from BER Level 1 group had significantly lower CAPS scores than those from Level 4 group, with the outcomes for those in Levels 2 and 3 falling in between. Outcomes did not change during followup; gains in each BER group were maintained.

The impact of categorisation based on the BER score was most evident in the SupC/EXP condition. At immediate post-treatment, participants in all groups showed equivalent improvement. However, by 6-month follow-up, there is a fan-like effect, where BER Level 1 group showed continuing improvement in CAPS score to 6-month follow-up, whereas the Level 4 group showed deterioration. At 6-month follow-up, the mean CAPS score for the BER Level 4 group was significantly worse than that for the BER Level 1 group.

The treatment effect moderator suggests which patients will benefit from a given treatment more than from another treatment. In this clinical trial, all patients are doing best in the STAIR/EXP treatment, where for participants at every level of BER, the best outcome at 6-month follow-up is achieved after STAIR/EXP treatment (Fig. 1). However, the moderator also provides information about the relative value of the other two treatment conditions. Comparison between STAIR/SupC and SupC/EXP (Fig. 1) indicated that participants in the lower levels of BER (Levels 1 and 2) have lower PTSD symptoms at 6-month follow-up in the exposure without skills (SupC/EXP) treatment than in the skills without exposure (STAIR/SupC) treatment. Conversely, participants at the higher BER levels (Levels 3 and 4) have better outcome at 6-month follow-up in the skills without exposure relative to the exposure and no skills treatment.

We compared the average CAPS PTSD scores at the 6-month follow-up among the following subgroups: (1) participants treated with skills and no exposure (STAIR/SupC), (2) participants treated with exposure and no skills (SupC/EXP); and (3) those who received their optimal treatment based on BER, i.e. participants at BER Levels 1 and 2 who received exposure without skills (SupC/EXP) and participants at BER Levels 3 and 4 who received skills without exposure. There were 23 participants whose actual treatment assignment matched what would have been assigned based on BER (13 in STAIR/SupC and 10 in SupC/EXP). At 6-month follow-up, the mean CAPS score for those who received their optimal treatment was 24.7, whereas that for participants treated with skills was $30.6(P=0.011)$ and the mean for those treated with exposure was $31.1 \quad(P=0.033)$, indicating better outcome if patients are assigned their treatment based on BER, than if everyone is treated with STAIR/SupC or SupC/EXP (see data supplement for details regarding how the test for the differences were conducted). Moreover, the impact of this difference should be interpreted in the context of considering the benefits as applied to an entire target population, where relatively small differences at the level of the individual have a large impact on social and economic well-being of the population as a whole. ${ }^{25}$

\section{Discussion}

PTSD predictor studies to date have reported inconsistent to nil results. We proposed that one reason for the absence of effects might be due to limited follow-up where outcomes have typically been assessed only immediately post-treatment. More importantly, recent reviews of moderation effects in clinical trials indicate that individual symptom measures are typically weak predictors of outcome. This study extended evaluation of treatment outcome to a 6-month follow-up and investigated whether a composite moderator of treatment outcome comprised of several measures would be a more sensitive predictor of differential outcome.

We identified an optimal linear combination of baseline patient characteristics, and constructed an interpretable GEM, symptom burden and emotion regulation (BER). BER included the total severity of all symptom measures (i.e. PTSD symptoms, depressive symptoms, dissociation and interpersonal difficulties) as well as the accumulation of identified positive characteristics, described as emotion regulation strength (i.e. anger management and general emotion regulation capacity). The resulting generated moderator score, BER, significantly predicted course of symptoms over time differentially across treatments (i.e. STAIR, exposure and a combination of the two treatments).

These findings are important for a number of reasons. The development of composite moderators has been recommended as a more powerful and precise measure of treatment outcome. ${ }^{23}$ This is the first study to utilise a composite moderator analysis to examine effect modifiers of PTSD treatment. Inconsistent results across studies to date may be explained by the relatively weak effects of individual measures tested as moderators of treatment outcome. The use of the traditional one-measure-at-a-time approach with the current sample produced null results similar to past studies, providing a demonstration of the differences in conclusion that can result depending on analytic strategy. The analyses also shed light on why trauma history (e.g. childhood abuse) sometimes but not always predicts outcome in treatment trials. It has been observed that early life and/or cumulative trauma is associated with increased symptom burden and impairment. However, history is actually only a general proxy for increasing symptom burden and problematic functioning. Individuals with similar history may vary on symptom severity and strengths depending on personal vulnerability (e.g. genetic load) and environmental (e.g. social support) factors. Given that interventions treat symptoms and not history, the use of symptom measures rather than history is likely to provide more relevant predictors of outcome and lead to more precise and personalised care.

The study included follow-up assessments at 3-and 6-month follow-up, unlike many previous treatment predictor studies. The moderator was not predictive of differential treatment outcome at immediate post-treatment, and variation in patient responses emerged only during follow-up. The results indicate the importance of including evaluation of patient status beyond the immediate conclusion of a psychotherapy trial, a time when patient response to treatment may be relatively uniform and generally positive. Time away from treatment and the emergence of new stressors and traumatic reminders can challenge the gains made during treatment. Last, our approach took into account both patient weaknesses and strengths. Although clinicians often consider patient strengths as well as psychopathology as they develop a treatment plan, this aspect of clinical care has not generally been translated into the research domain, particularly in the studies of PTSD treatment predictors.

It is frequently debated whether or not it is necessary for individuals with PTSD related to childhood abuse to receive enhanced interventions (e.g. skills training) to experience good 
treatment outcome. The data here suggest that skills training appears beneficial to all and may be necessary for a subset of vulnerable patients. Participants across all levels of BER had the best outcome in the STAIR/EXP intervention. Many individuals in exposure therapy without skills did well. However, participants with the highest BER scores (Level 4) showed deterioration during follow-up. The loss of benefits here, which is not seen in the other two groups, may be due to skills deficits that limit the ability to manage residual symptoms or to manage repeated or newly emerging life stressors which can exacerbate symptoms. Individuals with high BER would best be treated in either STAIR plus exposure therapy or, alternatively, STAIR without exposure. Indeed, our analyses indicated that among individuals who fell into either BER Level 3 or 4, skills training without exposure produced better outcome than exposure without skills. Still, it should be noted that among those with the lowest BER (Levels 1 and 2) treatment outcome was better in exposure therapy than in a skills only treatment.

These results demonstrate the contributing roles of cumulative symptom burden as well as of emotion regulation on influencing outcome. It also demonstrates how a moderator might function to provide measurement-based information to help guide patient and therapist decision-making about which treatment is expected to provide the greatest benefit to the individual patient. In the above analysis, for example, the results suggest that the combination treatment would be best. However, if the number of sessions or time for treatment was limited, then BER would help identify those who would do better in a brief course of skills training versus exposure therapy (i.e. those with high BER scores), those who would likely obtain greater benefits from exposure therapy versus skills training (i.e. those with low BER) and those for whom no difference in outcome is expected and thus could select their treatment based on personal preference (those with middle-level BER scores).

BER is a construct that is clinically intuitive and of treatment value. Although this research suggests the importance of assessing a range of symptoms as well as patient strengths, this is a demand that may be onerous in clinical practice. Future directions might include the reduction of measures contributing to combination moderators into a more manageable number of screening items and the development of online calculators for clinicians, which would allow a relatively rapid collection and analysis of information to guide treatment selection decisions. In addition to being patient-centred, this approach may help maximise resources by providing the treatment that the patient is statistically predicted to achieve the best results at the end of treatment and beyond.

Our results should be interpreted with certain cautions in mind. The sample size is small, and, accordingly, the results will need to be replicated. The sample consisted entirely of female participants who sought treatment for PTSD related to childhood abuse. Thus, it is unknown whether our findings would generalise to participants of other genders and those with other types of trauma histories (e.g. combat-related trauma). Composite moderators comprised of other measures might be more appropriate for other types of samples. For example, although the results from this study would suggest that both symptoms and strengths be taken into account, the type of symptoms used to measure burden might differ and the strengths identified might be other than emotion regulation capacities. Last, the construct needs further testing to determine whether it can inform clinical practice. The ultimate test for this and other treatment decision rules is to conduct and evaluate the outcome of clinical trials in which patients are randomised to conditions of treatment without use of the decision rule versus a condition in which the rule guides the selection of treatment.
In summary, this is the first study to utilise a composite moderator approach for PTSD treatments and demonstrates that interventions may differ in their benefits depending on patient characteristics. By implication, it suggests caution regarding the view that the routine application of a single treatment approach across all patients with PTSD is optimal care. This approach makes strides towards personalised medicine, efficiently incorporates multiple predictors in a statistically appropriate manner and may prove useful for clinicians for selecting optimal treatments for their patients in an empirical manner.

Marylene Cloitre, PhD, National Center for PTSD Dissemination and Training Division, VA Palo Alto Health Care System, Menlo Park, California, USA; NYU Langone Medical Center, New York, USA; Eva Petkova, PhD, Department of Child \& Adolescent Psychiatry, NYU Langone Medical Center; Nathan Kline Institute for Psychiatric Research, New York State Office of Mental HealthH, New York, New York, USA; Zhe Su, MA, Department of Child \& Adolescent Psychiatry, NYU Langone Medical Center, New York, New York, USA; Brandon J. Weiss, PhD, National Center for PTSD Dissemination and Training Division, VA Palo Alto Health Care System, Menlo Park, California, USA; Department of Psychiatry and Behavioral Sciences, Stanford University school of Medicine, Palo Alto, California, USA

Correspondence: Marylene Cloitre, National Center for PTSD Dissemination and Training Division, VA Palo Alto Health Care System, 795 Willow Road, Menlo Park, CA 94025, USA. Email: marylene.cloitre@va.gov

First received 20 Apr 2015, final revision 28 Jan 2016, accepted 29 Jan 2016

\section{Funding}

This study was supported by NIMH grants R01 MH86611 and R01 MH099003.

\section{References}

1 Kessler RC, Berglund $\mathrm{P}$, Demler $\mathrm{O}$, Jin R, Merikangas KR, Walters EE. Lifetime prevalence and age-of-onset distributions of DSM-IV disorders in the National Comorbidity Survey Replication. Arch Gen Psychiatry 2005; 62: 593-602.

2 American Psychiatric Association. Diagnostic and Statistical Manual of Mental Disorders, Fifth Edition (DSM-5). APA, 2013.

3 World Health Organization. The ICD-10 Classification of Mental and Behavioural Disorders: Clinical Descriptions and Diagnostic Guidelines. WHO, 1992.

4 Rodriguez P, Holowka DW, Marx BP. Assessment of posttraumatic stress disorderrelated functional impairment: a review. J Rehabil Res Dev 2012; 49: 649-65.

5 Schnurr PP, Lunney CA, Bovin MJ, Marx BP. Posttraumatic stress disorder and quality of life: extension of findings to veterans of the wars in Iraq and Afghanistan. Clin Psychol Rev 2009; 29: 727-35.

6 Australian Centre for Posttraumatic Mental Health. Australian Guidelines for the Treatment of Acute Stress Disorder and Post-traumatic Stress Disorder. ACPMH, 2013 (http://phoenixaustralia.org/wp-content/uploads/2015/03/Phoenix-ASD-PTSDGuidelines.pdf).

7 National Institute for Clinical Excellence. Post-Traumatic Stress Disorder (PTSD): The Management of PTSD in Adults and Children in Primary and Secondary Care (Clinical Guideline 26). NICE, 2005

8 Kessler RC, Sonnega A, Bromet E, Hughes M, Nelson CB. Posttraumatic stress disorder in the National Comorbidity Survey. Arch Gen Psychiatry 1995; 52: 1048-60.

9 Bradley R, Greene J, Russ E, Dutra L, Westen D. A multidimensional meta-analysis of psychotherapy for PTSD. Am J Psychiatry 2005; 162: 214-27.

10 Schottenbauer MA, Glass CR, Arnkoff DB, Tendick V, Gray SH. Nonresponse and dropout rates in outcome studies on PTSD: review and methodological considerations. Psychiatry 2008; 71: 134-68.

11 Kraemer HC, Wilson GT, Fairburn CG, Agras WS. Mediators and moderators of treatment effects in randomized clinical trials. Arch Gen Psychiatry 2002; 59: 877-83.

12 Institute of Medicine. Toward Precision Medicine: Building a Knowledge Network for Biomedical Research and a New Taxonomy of Disease. The National Academies Press, 2011.

13 Cloitre M, Courtois CA, Ford JD, Green BL, Alexander P, Briere J, et al. The ISTSS Expert Consensus Treatment Guidelines for Complex PTSD in Adults. International Society for Traumatic Stress Studies, 2012. 
14 Karam EG, Friedman MJ, Hill ED, Kessler RC, MCLaughlin KA, Petukhova M, et al. Cumulative traumas and risk thresholds: 12-month PTSD in the World Mental Health (WMH) surveys. Depress Anxiety 2014; 31: 130-42.

15 Taylor S, Fedoroff IC, Koch WJ, Thordarson DS, Fecteau G, Nicki RM. Posttraumatic stress disorder arising after road traffic collisions: patterns of response to cognitive-behavior therapy. J Consult Clin Psychol 2001; 69: 541-51.

16 Hagenaars MA, Van Minnen A, Hoogduin KA. The impact of dissociation and depression on the efficacy of prolonged exposure treatment for PTSD. Behav Res Ther 2010; 48: 19-27.

17 Van Minnen A, Arntz A, Keijsers GP. Prolonged exposure in patients with chronic PTSD: predictors of treatment outcome and dropout. Behav Res Ther 2002; 40 439-57.

18 Rizvi SL, Vogt DS, Resick PA. Cognitive and affective predictors of treatment outcome in Cognitive Processing Therapy and Prolonged Exposure for posttraumatic stress disorder. Behav Res Ther 2009; 47: 737-43.

19 Hembree EA, Street GP, Riggs DS, Foa EB. Do assault-related variables predic response to cognitive behavioral treatment for PTSD? J Consult Clin Psychol 2004; 72: $531-4$.

20 Resick PA, Suvak MK, Wells SY. The impact of childhood abuse among women with assault-related PTSD receiving short-term cognitive-behavioral therapy. I Trauma Stress 2014; 27: 558-67.

21 Van Minnen A, Harned MS, Zoellner L, Mills K. Examining potential contraindications for prolonged exposure therapy for PTSD. Eur J Psychotraumatol 2012 3: 1-241.

22 Bisson Jl, Roberts NP, Andrew M, Cooper R, Lewis C. Psychological therapies fo chronic post-traumatic stress disorder (PTSD) in adults. Cochrane Database Syst Rev 2013; 12: CD003388.

23 Wallace $M L$, Frank E, Kraemer HC. A novel approach for developing and interpreting treatment moderator profiles in randomized clinical trials. JAMA Psychiatry 2013; 70: 1241-7.

24 French B, Joo J, Geller NL, Kimmel SE, Rosenberg Y, Anderson JL, et al. Statistical design of personalized medicine interventions: the Clarification of Optimal Anticoagulation through Genetics (COAG) trial. Trials 2010; 11: 108.
25 Murphy SA. Optimal dynamic treatment regimes. J Roy Stat Soc B 2003; 58: 331-66.

26 Petkova E, Su Z. Modeling strategies for developing treatment responses indices. (Session IPS021). In Proceedings of the 59th ISI World Statistics Conference, 25-30 August 2013, Hong Kong: 121-5. Avaialable at http://www.statistics.gov.hk/wsc/ IPS012-P3-S.pdf (accessed 28 Jan 2016).

27 Cloitre $M$, Stovall-McClough $K C$, Nooner $K$, Zorbas $P$, Cherry $S$, Jackson $C L$, et al. Treatment for PTSD related to childhood abuse: a randomized controlled trial. Am J Psychiatry 2010; 167: 915-24.

28 Weathers FW, Keane TM, Davidson JR. Clinician-administered PTSD scale: a review of the first ten years of research. Depress Anxiety 2001; 13: 132-56.

29 Beck AT, Steer RA, Brown GK. Manuel for the Beck Depression Inventory-II. Psychological Corporation, 1996.

30 Briere J. Trauma Symptom Inventory Professional Manual. Psychological Assessment Resources, 1995.

31 Horowitz LM, Rosenberg SE, Baer BA, Ureño G, Villaseñor VS. Inventory of interpersonal problems: psychometric properties and clinical applications. J Consult Clin Psychol 1988; 56: 885-92.

32 Spielberger C. State-Trait Anger Expression Inventory Manual. Psychological Assessment Resources, 1991.

33 Catanzaro SJ, Mearns J. Measuring generalized expectancies for negative mood regulation: initial scale development and implications. J Pers Assess 1990; 54: $546-63$.

34 Diggle PJ, Heagerty P, Liang K-Y, Zeger SL. Analysis of Longitudinal Data (2nd edn). Oxford University Press, 2002.

35 R Core Team. R: A Language and Environment for Statistical Computing. R Foundation for Statistical Computing, 2013

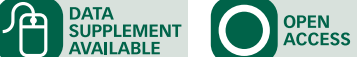

